\title{
Correlation of Dysmenorrhea and Lower Back Pain among Young Female Students - A Cross-Sectional Study
}

WAJEEHA WAHID ${ }^{1}$, MUHAMMAD ASIF ${ }^{2}$, JANSHER MANSOORI ${ }^{3}$, MUHAMMAD HUSSAIN IQBAL ${ }^{4}$, FARIHA KHALID ${ }^{5}$

${ }^{1}$ Student at University Institute of Physical Therapy (University of Lahore)

${ }^{2,4,5}$ Senior Lecturer at University Institute of Physical Therapy (University of Lahore)

${ }^{3} \mathrm{HOD}$ Physiotherapy Department at Civil Hospital Daska

Correspondence to Wajeeha Wahid, Email: Wakeupwajeeha98@gmail.com

\begin{abstract}
Aim: The aim of this study is to determine the Correlation of dysmenorrhea and lower back pain among young female student. Study design: A cross sectional study design is used.

Methods: After meeting the criteria of 165 female students the data will collected from different public and private universities and colleges in Lahore such as UOL, UMT, KIPS, UCP and the Performa for this purpose will attach here with Convenient sampling will be used by using different questionnaire to gather data from female students to take consent to further proceed this data surely, your privacy will not be consented private.

Results: The histogram with normal curve for Age showed that mean and standard deviation found to be $20.76+3.366$ while curve was normally distributed. The results regarding back pain showed that there were $46.1 \%$ yes and $53.9 \%$ no. The results regarding intensity of back pain showed that there were $12.1 \%$ no pain, $10.9 \% 1,8.5 \% 2,7.9 \% 3,7.9 \% 4,8.5 \% 5,9.1 \% 6,10.3 \%$ $7,6.7 \% 8,10.3 \% 9$, and $7.9 \%$ worst pain. The results regarding socio economics showed that there were $32.7 \%$ upper $35.2 \%$ middle and $32.1 \%$ lower.

Conclusion: There is positive correlation between dysmenorrhea and lower back pain. Many young females suffer from dysmenorrhea and their symptoms related to back pain. It also affect their working ability.

Keywords: Correlation, Dysmenorrhea, Lower Back Pain, Young Females Students.
\end{abstract}

\section{INTRODUCTION}

Females of reproductive age often suffer from dysmenorrhea, a painful condition. Despite dysmenorrhea being generally considered a benign condition, a significant disability can result from it. Researchers suggest that dysmenorrhea has been linked to migraines, headaches, and fibromyalgia, among other painful disorders. The authors reported further evidence that dysmenorrhea can alter noxious sensory processing ${ }^{1}$.

Female fertility is influenced by several components of the menstrual cycle including the menstrual period, one of the phases of the menstrual cycle. Dysmenorrhea is characterized as pain and soreness in the lower abdomen or lower back. There are two types of dysmenorrhea: primary and secondary ${ }^{2}$.

Primary dysmenorrhea is discomfort that occurs before or during menstruation and is unrelated to other disorders and among young women, is the most prevalent gynecological problem; secondary - pain produced by a condition in the woman's reproductive organs, such as endometriosis, amenorrhea, ovarian cysts, or infection during menstruation ${ }^{3}$.

Dysmenorrhea is most common in adolescent girls and young women, with a prevalence of $70 \%$ to $90 \%$, and can have a considerable impact on a woman's quality of life. Menstrual pain usually stops after childbirth due to a shift in hormonal balance ${ }^{4}$. The pain can start before or after monthly bleeding begins, and it normally lasts for several hours to two days. It's usually a nonspecific discomfort that's hard to pinpoint and much harder to express. It can also be felt in the surrounding organs' bones, muscles, and skin. The pain is minor and bearable in the some women, but it is moderate or severe in others, and it is primarily centered in the lower abdomen. Nausea, vomiting, nonspecific abdominal discomfort, breast tenderness, vaginal swelling, and diarrhea are all possible side effects. Furthermore, discomfort can be felt not only in the abdomen but also in the head, neck, lower back, pelvis, and thighs in certain women.

Dysmenorrhea sufferers were more likely than nondysmenorrhea sufferers to report mood changes. Women rarely seek medical attention for this problem, even though it's very common $^{5}$. The specific etiology of the symptoms of dysmenorrhea

Received on 17-05-2021

Accepted on 27-09-2021 is unknown. Some studies link the symptoms to uterine prostaglandin activities, specifically PGF2a. It has been identified that dysmenorrhea is caused by an excess of vasopressin, a hormone that constricts muscle tissue ${ }^{6}$.

There have also been studies linking these symptoms to the body's musculoskeletal system. According to one idea, there is a link between abnormal pelvic posture, lumbar vertebrae, and stomach muscular spasms, all of which alter the position of the uterus, increasing the risk of dysmenorrhea. By producing vasoconstriction, the location of the lumbar vertebrae can also impact the blood flow to the uterus, resulting in pain. Another possibility is that the musculoskeletal system is also influenced by hormonal factors during the menstrual cycle, which can cause discomfort ${ }^{7}$.

According to previous research looking into the link between menstruation pain and musculoskeletal pain, women who had menstrual pain that was a 3 on the Visual Analog Scale (VAS) had higher musculoskeletal discomfort ${ }^{8}$.

\section{METHODOLOGY}

After meeting the criteria of 165 female students and permission from Ethical Review Board, the data was collected from different public and private universities and colleges in Lahore and the Performa for this purpose was attached here with Convenient sampling was used by using different questionnaire to gather data from female students to take consent to further proceed this data surely, your privacy would not be consented private.

\section{RESULTS}

The histogram with normal curve for Age showed that mean and standard deviation found to be $20.76+3.366$ while curve was normally distributed. The results regarding back pain showed that there were $46.1 \%$ yes and $53.9 \%$ no. The results regarding intensity of back pain showed that there were $12.1 \%$ no pain, $10.9 \%$ $1,8.5 \% 2,7.9 \% 3,7.9 \%$ 4, 8.5\% 5, 9.1\% 6, 10.3\% 7, 6.7\% 8, $10.3 \%$ 9 , and $7.9 \%$ worst pain. The results regarding socioeconomic showed that there were $32.7 \%$ upper $35.2 \%$ middle and $32.1 \%$ lower. The results regarding family history of dysmenorrhea showed that there were $50.9 \%$ yes and $49.1 \%$ no. The results regarding age of menarche year showed that there were $37 \%$ less 
than $12,31.5 \% 13-14$ and $31.5 \%$ greater than 15 . The results regarding coffee consumption showed that there were $52.1 \%$ yes and $47.9 \%$ no. The results regarding dysmenorrhea showed that there were $31.5 \%$ Menstruation is not painful and daily activity, $20.6 \%$ Menstruation is painful but seldom inhibits required normal activity; analgesics are seldom required, $24.8 \%$ Daily activity is affected; analgesics required and give sufficient relief so to avoid absence and $23.0 \%$ Activity clearly inhibited; poor effect of analgesics vegetative symptoms (headache, fatigue, vomiting, and diarrhea). The results regarding working ability showed that there were $24.8 \%$ unaffected, $21.2 \%$ rarely affected, $29.1 \%$ moderately affected, and $24.8 \%$ clearly inhibited. The results regarding systemic symptoms showed that there were $27.9 \%$ none, $20.6 \%$ mild, $19.4 \%$ few, and $32.1 \%$ apparent. The results regarding analgesics showed that there were $23.6 \%$ not required, $26.1 \%$ rarely required, $26.1 \%$ required, and $24.2 \%$ poor effect.

Table 1: The results regarding back pain showed that there were $46.1 \%$ yes and $53.9 \%$ no.

\section{Back pain}

\begin{tabular}{|l|c|c|c|c|}
\hline Valid & Frequency & $\%$ & Valid\% & Cumulative\% \\
\hline Yes & 76 & 46.1 & 46.1 & 46.1 \\
\hline No & 89 & 53.9 & 53.9 & 100.0 \\
\hline Total & 165 & 100.0 & 100.0 & \\
\hline
\end{tabular}

Table 2: The results regarding intensity of back pain showed that there were $12.1 \%$ no pain, $10.9 \% 1,8.5 \% 2,7.9 \% 3,7.9 \% 4,8.5 \%$ $5,9.1 \% 6,10.3 \% 7,6.7 \% 8,10.3 \% 9$, and $7.9 \%$ worst pain.

Intensity of back pain

\begin{tabular}{|l|c|c|c|c|}
\hline Valid & Frequency & $\%$ & Valid\% & Cumulative\% \\
\hline No pain & 20 & 12.1 & 12.1 & 12.1 \\
\hline 1 & 18 & 10.9 & 10.9 & 23.0 \\
\hline 2 & 14 & 8.5 & 8.5 & 31.5 \\
\hline 3 & 13 & 7.9 & 7.9 & 39.4 \\
\hline 4 & 13 & 7.9 & 7.9 & 47.3 \\
\hline 5 & 14 & 8.5 & 8.5 & 55.8 \\
\hline 6 & 15 & 9.1 & 9.1 & 64.8 \\
\hline 7 & 17 & 10.3 & 10.3 & 75.2 \\
\hline 8 & 11 & 6.7 & 6.7 & 81.8 \\
\hline 9 & 17 & 10.3 & 10.3 & 92.1 \\
\hline Worst pain & 13 & 7.9 & 7.9 & 100.0 \\
\hline Total & 165 & 100.0 & 100.0 & \\
\hline
\end{tabular}

Table3: The results regarding socioeconomics showed that there were $32.7 \%$ upper $35.2 \%$ middle and $32.1 \%$ lower.

Socioeconomics

\begin{tabular}{|l|c|c|c|c|}
\hline Valid & Frequency & $\%$ & Valid\% & Cumulative\% \\
\hline Upper & 54 & 32.7 & 32.7 & 32.7 \\
\hline Middle & 58 & 35.2 & 35.2 & 67.9 \\
\hline Lower & 53 & 32.1 & 32.1 & 100.0 \\
\hline Total & 165 & 100.0 & 100.0 & \\
\hline
\end{tabular}

Table 4: The results regarding family history of dysmenorrhea showed that there were $50.9 \%$ yes and $49.1 \%$ no.

Family history of dysmenorrhea

\begin{tabular}{|l|c|c|c|c|}
\hline Valid & Frequency & $\%$ & Valid\% & Cumulative\% \\
\hline Yes & 84 & 50.9 & 50.9 & 50.9 \\
\hline No & 81 & 49.1 & 49.1 & 100.0 \\
\hline Total & 165 & 100.0 & 100.0 & \\
\hline
\end{tabular}

\section{DISCUSSION}

Adolescent girls are most vulnerable to dysmenorrhea, a gynecological complaint. It has a negative impact on the academic, participation in sports, and peer networking ${ }^{9}$. According to reports, women experience dysmenorrhea between $60 \%$ and $93 \%$ of the time ${ }^{10}$. Women often experience premenstrual symptoms as well as dysmenorrhea ${ }^{11}$.

Overall, the relationship between social support and reporting menstrual pain was not significant. However, a more indepth examination of the components of social support highlighted the significance of social support loss. Over the previous year, women with greater disruptions in their social networks had more menstruation symptoms than those with steady support ${ }^{12}$.

A previous study found a link between primary dysmenorrhea and mood problems. Gulsah Balk et al. (2014) discovered a link between dysmenorrhea and mood disorders. Dysmenorrhea in adolescent girls is associated with an increased incidence of sadness and anxiety. The findings of their study are crucial in stressing the significance of a multidisciplinary approach to primary dysmenorrhea treatment and follow-up. More research into dysmenorrhea and psychiatric illnesses will aid in developing an effective treatment for this common adolescent girl's condition ${ }^{8}$.

In this current study I wanted to determine the correlation of dysmenorrhea and lower back pain among young female students and the results were satisfying. Young females suffering from dysmenorrhea have many symptoms including severe back pain. Symptoms of dysmenorrhea affect the working abilities of female. More researchers has to be done for their treatment.

\section{CONCLUSION}

There is positive correlation between dysmenorrhea and lower back pain. Many young females suffer from dysmenorrhea and their symptoms related to back pain. It also affect their working ability.

\section{Conflict of interest: Nil}

\section{REFERENCES}

1. Archer DF. Menstrual-cycle-related symptoms: a review of the rationale for continuous use of oral contraceptives. Contraception. 2006;74(5):359-66.

2. Tu C-H, Niddam DM, Chao H-T, Chen L-F, Chen Y-S, Wu Y-T, et al. Brain morphological changes associated with cyclic menstrual pain. Pain. 2010;150(3):462-8.

3. Pan Y-Y, Jin S-H, Luo S, Li R-R, Tu Y-Y, Jin D, et al. Dysmenorrhea is associated with a higher incidence of pain after diagnostic hysteroscopy or treatment. Signa Vitae. 2021;17(2):63-6.

4. Chandrima FA. A survey on knowledge and awareness of dysmenorrhea among the female students of East West University: East West University; 2017.

5. 이지산. Method of App Selection based on Users Needs (MASUN): Randomized Controlled Trial of Dysmenorrhea and PMS: 서울대학교 대학원; 2018.

6. Lumsden M. Prostaglandins in dysmenorrhoea and endometriosis. Prostaglandins and the Uterus: Springer; 1992. p. 45-66.

7. Sultan C, Gaspari L, Paris F. Adolescent dysmenorrhea. Pediatric and adolescent gynecology. 2012;22:171-80.

8. Balık G, Üstüner I, Kağıtcı M, Şahin FK. Is there a relationship between mood disorders and dysmenorrhea? Journal of pediatric and adolescent gynecology. 2014;27(6):371-4

9. Harlow BL, Cohen LS, Otto MW, Spiegelman D, Cramer DW. Early life menstrual characteristics and pregnancy experiences among women with and without major depression: the Harvard study of moods and cycles. Journal of Affective Disorders. 2004;79(1-3):16776.

10. Wong LP, Khoo EM. Dysmenorrhea in a multiethnic population of adolescent Asian girls. International Journal of Gynecology \& Obstetrics. 2010;108(2):139-42.

11. Kitamura M, Takeda T, Koga S, Nagase S, Yaegashi N. Relationship between premenstrual symptoms and dysmenorrhea in Japanese high school students. Archives of women's mental health. 2012;15(2):131-3.

12. Alonso C, Coe CL. Disruptions of social relationships accentuate the association between emotional distress and menstrual pain in young women. Health Psychology. 2001;20(6):411 